\title{
An award to oxygen sensing
}

\author{
The discoveries of this year's Nobel laureates in Physiology and Medicine have improved diagnostic and \\ therapeutic strategies relying on oxygen sensing.
}

T he 2019 Nobel Prize in Physiology and Medicine - jointly awarded to William Kaelin, Jr., Sir Peter Ratcliffe and Gregg Semenza - recognizes the body of work describing the mechanism of "how cells sense and adapt to oxygen availability". Changes in oxygen availability are sensed by the protein hypoxia inducible factor- $1 \alpha$ (HIF-1 $\alpha$; discovered by Semenza), whose oxygen-dependent degradation is regulated by the Von Hippel Lindau tumoursuppressor protein (VHL; described by Kaelin and Ratcliffe). In hypoxic conditions, VHL inhibits the degradation of HIF- $1 \alpha$, allowing more of the protein to translocate to the cell's nucleus, where it acts as a transcription factor, upregulating (often within minutes) the production of proteins (such as the vascular endothelial growth factor, erythropoietin and the inducible nitric oxide synthase) that stimulate blood perfusion in tissues and thus tissue oxygenation. The award comes 88 years after Otto Warburg won the same Nobel for his discovery "of the nature and mode of action of the respiratory enzyme", which he investigated after observing differences in oxygen consumption between cancer cells and normal cells (J. Gen. Physiol. 8, 519-530; 1927, Science 68, 437-443; 1928); and 66 years after his student, Hans Krebs, won the award alongside Fritz Lipmann in 1953, for his discovery "of the citric acid cycle" - which explains the release of stored energy by cells through the oxidation (into adenosine triphosphate and carbon dioxide) of the acetyl form of coenzyme A (discovered by Lipmann) formed by the breakdown of carbohydrates and fatty acids.

Warburg found that cancer cells have defective mitochondria and dysfunctional respiration, and that they display an increased dependence on glucose and glycolysis as a source of energy (even at atmospheric oxygen pressure). This phenomenon, known as aerobic glycolysis, led Warburg to postulate that mitochondrial dysfunction caused cancer, a proposition that has long been disproved. This year's Nobel Prize acknowledges that the adaptation of cells to variable oxygen levels informs our understanding of oncogenic transformation, and of a range of phenomena in normal physiology and development.
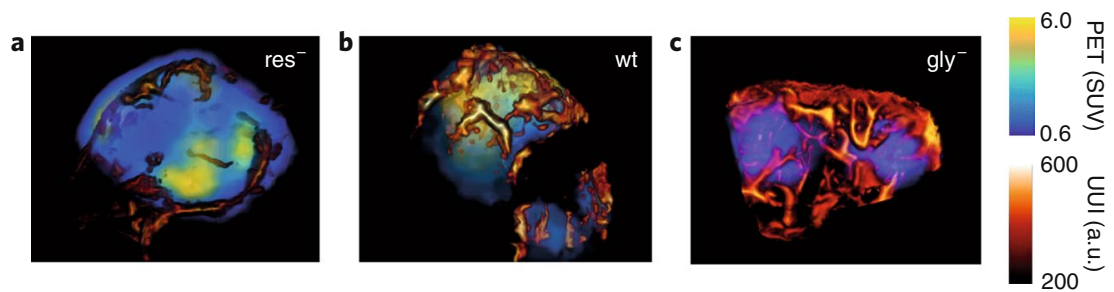

Fig. 1 | In vivo imaging relating glucose metabolism with the vascular network density of subcutaneous tumour xenografts via FDG-PET and ultrafast ultrasound. Respiration-deficient (res- ${ }^{-}$a) tumours display higher glucose metabolism and lower vessel density than wild-type (wt, b) tumours, whose metabolic activity is in turn higher than those of glycolysis-deficient $\left(\mathrm{gly}^{-}, \mathbf{c}\right)$ tumours, which have higher vessel density. Tumour vasculature appears in red, and high metabolic activity is displayed in yellow and green. SUV, standardized uptake value. Figure reproduced from Provost, J. Nat. Biomed. Eng. 2, 85-94 (2018).

This is the case for tumour hypoxia - a hallmark of cancer cells that is physiologically associated with the disorganised and tortuous vasculature resulting from the expanding tumour mass, which exerts localized mechanical pressure and induces the formation of new vessels. The dysregulated vasculature primarily causes a limited supply of nutrients (especially oxygen), and the ineffective removal of cellular waste products (in particular, carbon dioxide and lactate). In contrast, normal cells exposed to a rapid increase in metabolism, in the absence of a sufficient supply of oxygen, revert to glucose as a source of energy (known as anaerobic glycolysis). By modulating oxygen levels in cancer cells, differences between aerobic and anaerobic metabolism can be easily imaged and studied. Furthermore, by tracking blood-oxygenation levels, the macroscopic features of the vasculature can be linked to the hypoxic phenotype (Fig. 1). This knowledge allows the spatial and dynamic heterogeneity of vascular perfusion in a tumour to be mapped to, for example, predict drug uptake and treatment response.

Hypoxic cells, which rely on glycolysis, cause the progressive acidification of the tumour microenvironment. Research on how cancer cells survive low-oxygen and acidic environments and drive neoangiogenesis led to the discovery of the 'lactate shuttle' (whereby adjacent cells adopt differing metabolic pathways, sharing metabolic by-products) and of the phenomenon of cycling (or intermittent) hypoxia, which causes the transcriptional upregulation of HIF- $1 \alpha$ by the nuclear factor kappa-b), as well as to the development of tracers for imaging $\mathrm{pH}$ and other intermediate glycolytic metabolites. The most common positron emission tomography (PET) tracer - the glucose analogue ${ }^{18} \mathrm{~F}-\mathrm{FDG}$ (2-deoxy-2-[18F]fluoro-d-glucose), whose uptake is dependent on the expression of proteins that fall under the transcriptional control of HIF- $1 \alpha-$ is an indirect measurement of metabolic changes between aerobic and anaerobic glycolytic states (Fig. 1). The acidic milieu of small tumours can also be detected via $\mathrm{pH}$-sensitive positron-emitting polycationic polymers and via activatable polymer-conjugated iridium complexes.

Oxygen sensing can be used for diagnostics because blood-oxygen levels can be detected via the spectroscopic differences between oxygenated and deoxygenated haemoglobin. For example, haemodynamics can be imaged via optical coherence tomography to monitor ischaemic brain tissue in patients suffering from stroke, and ischaemic brain injury in infants with congenital heart defects undergoing heart surgery. And, because HIF- $1 \alpha$ also regulates vascular radiosensitivity in tumours, haemodynamics can be imaged to determine the delivery of radiation therapy. Pulse oximeters for the monitoring of cardiac function, and photoacoustic imaging for imaging angiogenesis, also measure oxygenation levels. Blood-oxygenation-leveldependent magnetic resonance imaging 
(known as BOLD-MRI), a functional MRI technique for imaging blood flow in the brain, relies on the paramagnetic difference between oxygenated and deoxygenated haemoglobin, and its signal correlates with the expression of HIF-1 $\alpha$. Systemically injected radiolabelled probes (such as ${ }^{18} \mathrm{~F}$-labelled fluoromisonidazole) for PET can also selectively bind to macromolecules in hypoxic cells. These techniques are clinically used to monitor the pathophysiological consequences of HIF- $1 \alpha$ activation, such as changes in the hypoxic fraction, or vascularization following therapy.

The understanding of metabolic adaptation in cells, enabled by the discoveries of this year's Nobel laureates, has also led to the design of therapeutic strategies for anaemia, cartilage repair and wound healing, and facilitated biomedical applications such as the characterization of intratumoral metabolic heterogeneity. Visualizing how cells sense and adapt to oxygen availability thus helps the design of strategies for the detection of, and intervention in, the underlying causes of metabolic dysregulation, for example in improving the efficacy of photodynamic therapy via the generation of reactive oxygen species in the hypoxic areas of a tumour. Much is thus owed to the new Nobel laureates and to the many researchers who have built on their efforts.

Published online: 18 October 2019 https://doi.org/10.1038/s41551-019-0479-z 